Thorax (1952), 7, 182.

\title{
A CASE OF PULMONARY CRYPTOCOCCOSIS
}

BY

\author{
D. BARRON CRUICKSHANK AND G. KENT HARRISON \\ From the Pathological and Surgical Departments, Papworth Sanatorium, Cambridge
}

(RECEIVED FOR PUBLICATION NOVEMBER 20, 1951)

The patient, a married woman of 50 , with no extensive travel outside East Anglia, had an attack of influenza in January, 1951, which left her with a cough which persisted up to the time of operation in August, 1951. There was only a trace of sputum and no haemoptysis or pain, and apart from losing 1 st. in weight over the previous six months and a slightly unhealthy appearance, she was otherwise normal and fit. She was referred to the chest physician (Dr. C. Downes), and a radiograph taken on June 7, 1951, revealed, as the only abnormality, an opacity occupying a position in the right middle and anterior portion of the right lower lobe. She was bronchoscoped on June 13, and a rounded mass was seen just inside the orifice of the middle lobe bronchus. The bronchial aspirate contained numerous morphologically typical Cryptococci neoformans, and these were also present in the bronchial biopsy. Aerobic cultures of the aspirate yielded numerous culturally typical, yeast-like colonies growing preferentially on Sabouraud's medium and at room temperature, and more reluctantly on blood agar and at $37^{\circ} \mathrm{C}$. (Kurung, 1942). A guinea-pig inoculated with the aspirate died 24 days later with gastroenteritis and yielded positive cryptococcal cultures from spleen and peritoneum. A mouse injected with a heaviy subculture survived and was killed at seven weeks while its only gross pathology was a few foci in the live (histologically cryptococcal), all the organs yielded positive cryptococcal cultures (liver, spleen, kidney, lung, brain, and peritoneum).

The cryptococcus strain was insensitive to penicilla streptomycin, aureomycin, sulphathiazole, sulphadiazing, sulphamerazine, and sulphamezathine. There wege no cerebral symptoms and the lumbar C.S.F. was normal. A right pneumonectomy was successfu用 performed on June 26. The incised pneumonecton specimen revealed a tumour-like mass occupying tofe middle lobe, which was firm, with a diffuse extensiog to the basal segments of the lower lobe, which wope doughy. The upper lobe was unaffected and there was no enlargement of the regional glands. Histologica的 the " tumour" and extension consisted of a solid mass of cryptococci replacing the parenchyma and practica devoid of any supporting stroma; it was surrounded By a fibrous capsule in which appeared lymphocytes amed giant cells.

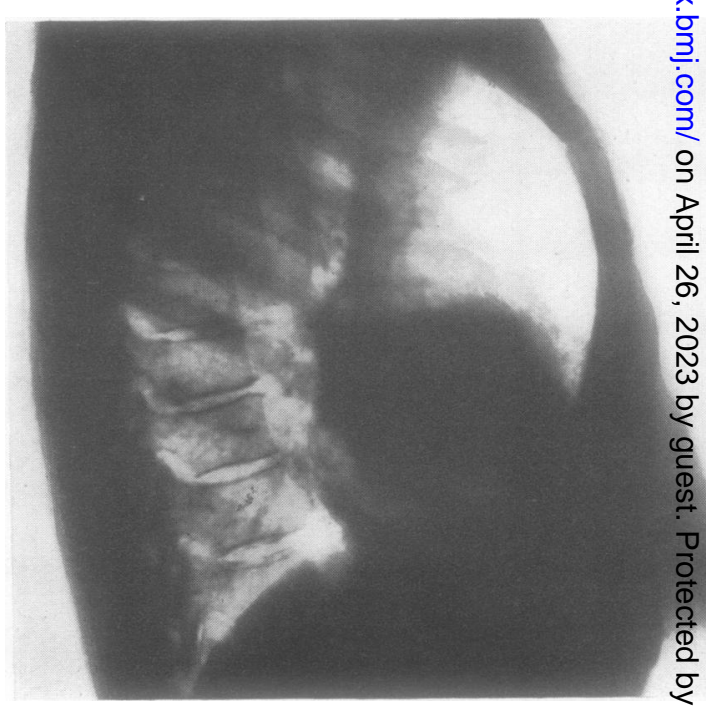

Fig. 2

TiG. 1
Figs. 1 and 2.-Postero-anterior and lateral films of June 22, 1951. 


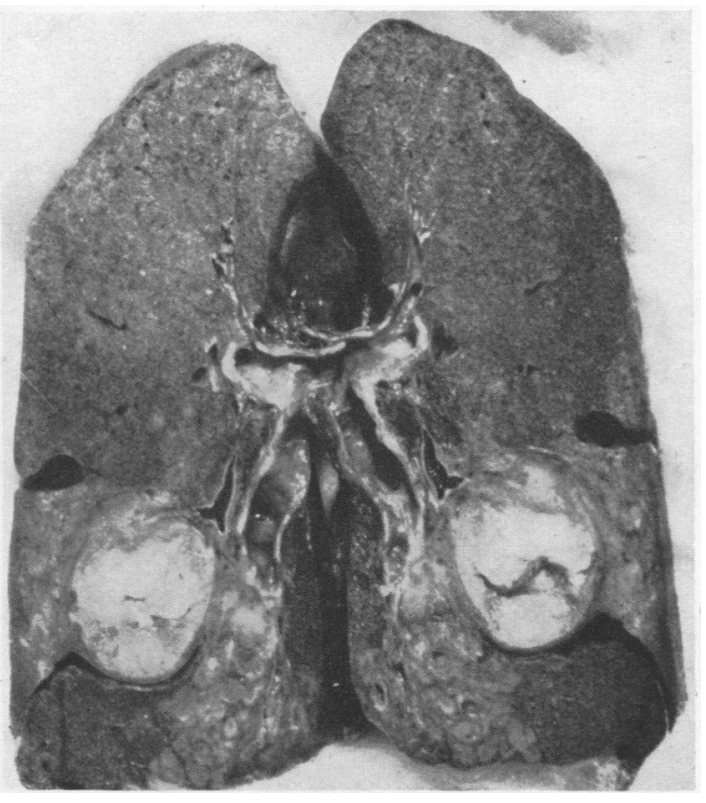

Fig. 3

Six days after operation the patient died suddenly from embolism of the left pulmonary artery. Before making the necropsy C.S.F. was removed by cisternal puncture; this fluid was blood-stained and loaded with cryptoocci. At necropsy the brain showed merely some regions of indefinite degeneration (e.g., right third

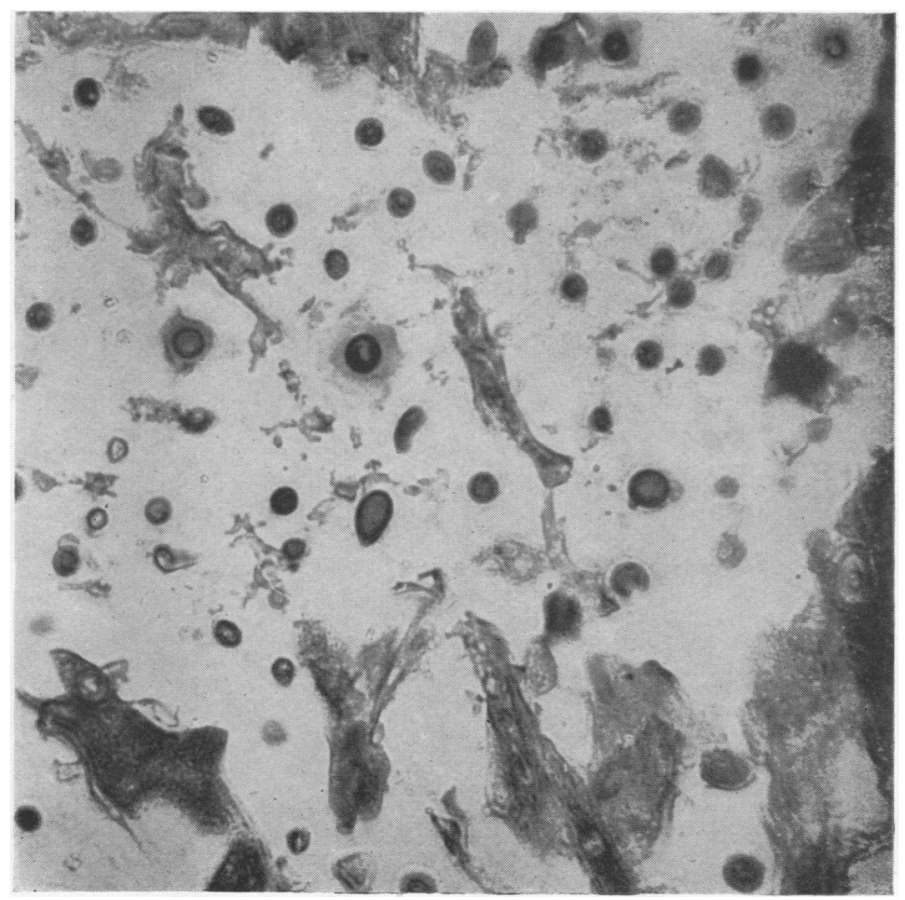

Fig. 4
FIG. 3.-Incised specimen showing toruloma in middle lobe which bulges into oblique fissure with diffuse spread to the lower lobe.

FIG. 4.-Section from centre of toruloma: juxtaposed cryptococci appear widely spaced because of their broad, invisible mucoid capsules. MassonMallory (1/6 in objective)

FIG. 5.-Incised specimen cut to show involvement of the anterior basal segment of the lower lobe.

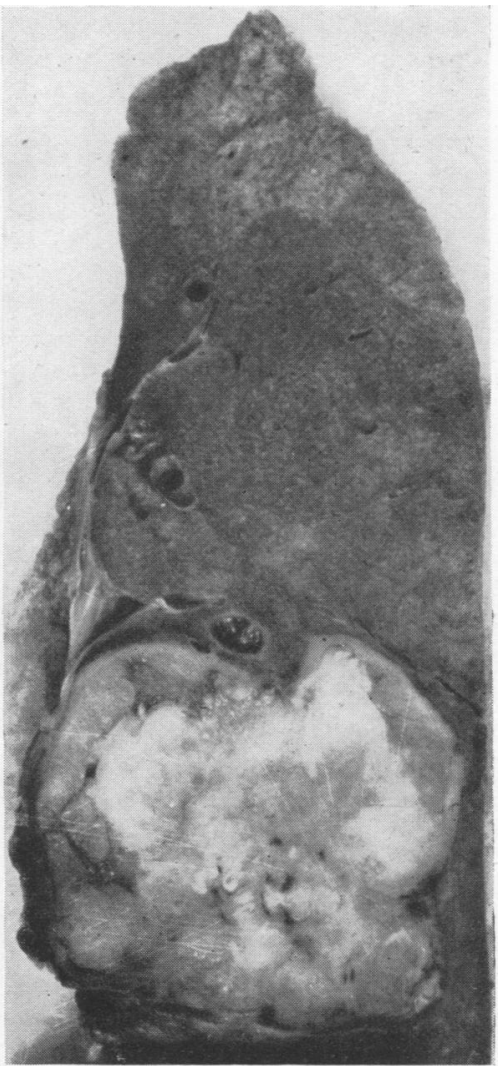

Fig. 5

ventricle) and it required extensive histology to demonstrate the presence of cryptococci in the subarachnoid layers of the medulla, the only specific abnormality found. Other organs showed nothing significant. No cultures were prepared from the necropsy material.

\section{Discussion}

Cryptococcosis is not endemic in Great Britain, only some half-dozen cases having been previously described (Carter and Young, 1950; Daniel, Schiller, and Vollum, 1949). In the lung the disease may be tumour-like (" toruloma "), bronchopneumonic, or miliary; it is usually progressive and ultimately initiates a fatal meningitis. This predilection for the brain and meninges $(60 \%)$ and lung $(20 \%)$ (Levin, 1937), taken in conjunction with symptomatology, necessitates differentiation from tuberculous meningitis, tuberculoma, or pulmonary tuberculosis (Hobby, 1949). Indeed, as 
cerebral involvement is usually preceded by some pulmonary symptoms, the lung is regarded as the usual portal of entry (Krainer, Small, Hewlitt, and Deness, 1946). The disease is highly lethal, affects people of all ages, and women about twice as frequently as men. Chemotherapy is disappointing (Hamilton and Thompson, 1946), and as the broad gelatinous capsule prevents antibody formation vaccine therapy also fails (Benham, 1935). Two successful pneumonectomies have been reported (Froio and Bailey, 1949; Dormer, Friedlander, Wiles, and Simson, 1945).

The source of the fungus is equivocal; in endemic areas a strain can be recovered from the faeces of certain animals prone to natural cryptococcal infections and from fermenting fruit substrates; the fruit strain is pathogenic to these animals, but both animal and fruit strains are serologically distinct from those which occur in man, and are nonpathogenic to man. In man two strains occur, skin saprophytes and true pathogens; these are morphologically identical and serologically related, and can only be distinguished by virulence tests in animals. Because of these complex facts autogenous infection is premised, i.e., that the skin saprophyte acquires pathogenicity (Conant, Martin, Smith, Baker, and Callaway, 1944). Despite an extens Fe literature (over 100 cases have been described siree the first recorded case in 1894), the only known castes of direct infections in man are three prematioe infants who contracted fatal cryptococcal menings;encephalitis from cryptococci present in the materngl genital tract (Neuhauser and Tucker, 1948).

\section{SUMMARY}

A case of pulmonary cryptococcosis is recorded. REFERENCES

Benham, R. W. (1935). J. infect. Dis., 57, 255.

Carter, H. S., and Young, J. L. (1950). J. Path. Bact., 62, 271. N Conant, N. F., Martin, D. S., Smith, D. T., Baker, R. D., and Collaway, J. L. (1944). Manual of Clinical Mycology, p. 11 Military Medical Manuals: National Research Council (prepared under auspices of the Division of Medical Sciences of the National Research Council) Saunders. Philadelphia.

Daniel, P. M. Schiller, F., and Vollum, R. L. (1949). Lancet, 1,

Dormer, B. A., Friedlander, J., Wiles, F. J., and Simson, F. QVV (1945). J. thorac. Surg., 14, 322.

Froio, G. F., and Bailey, C. P. (1949). Dis. Chest, 16, 354. Hamilton, L. C., and Thompson, P. E. (1946). Amer. J. Dis. Ch

Hobby, A. W. (1949). Dis. Chest, 15, 182.

Krainer, L., Small, J. M., Hewlitt, A. B., and Deness, T. (1986) J. Neurol. Neurosurg. Psychiat., 9, 158.

Kurung, J. M. (1942). Amer. Rev. Tuberc., 46, 365.

Levin, E. A. (1937). Arch. intern. Med., 59, 667.

Neuhauser, E. B. D., and Tucker, A. (1948). Amer. J. Roentger 59,805 . 